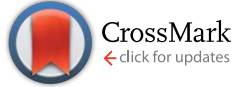

Cite this: RSC Adv., 2016, 6, 55736
Received 28th January 2016

Accepted 4th June 2016

DOI: $10.1039 / \mathrm{c} 6 \mathrm{ra02620f}$

www.rsc.org/advances

\section{Full alignment of dispersed colloidal nanorods by alternating electric fields $\uparrow$}

\author{
M. Mohammadimasoudi, ${ }^{* a c}$ Z. Hens ${ }^{\mathrm{bc}}$ and K. Neyts ${ }^{* a c}$
}

The parallel alignment of an ensemble of colloidal nanorods may unleash their application as the optically anisotropic constituent in polarized fluorescent sheets or polarization-selective detectors. Here, we demonstrate that full alignment of colloidal CdSe/CdS nanorods in suspension can be achieved by applying AC electric fields. Alignment is monitored by the concurrent change of the optical transmission of the dispersion. By comparing the transmission measurements to a theoretical model encompassing both the permanent and induced dipole moments of the nanorods, we can attribute the alignment to the interaction between the electric field and the nanorod's permanent dipole moment. The permanent dipole moment, relaxation time, absorption anisotropy and critical frequency of the CdSe/CdS nanorods are determined. In addition, we show that the regime of full alignment enables the direct determination of the anisotropic absorption of CdSe/CdS nanorods. We find that the anisotropy in absorption for the CdSe dot is similar to that of the CdS rod, which we attribute to the similarity in dielectric constant and electric field in both materials.

\section{Introduction}

Over the last 30 years, colloidal nanocrystals have attracted wide scientific and technological interest since their opto-electronic properties can be tuned by means of their size and shape. Whereas size is the key parameter underlying quantization in semiconductor nanocrystals ${ }^{1}$ and the occurrence of localized surface plasmon resonances in metal nanocrystals, ${ }^{2}$ shape often adds unique possibilities to further adjust the nanocrystal properties. $^{3}$ In this respect, rods and platelets stand out since their shape anisotropy results in an anisotropic interaction absorption, emission or scattering - with light. ${ }^{4}$ Metallic nanorods, for example, exhibit a splitting of the localized surface plasmon resonance into a longitudinal and transversal mode that most strongly interacts with light polarized perpendicular or parallel to the long axis of the rod, respectively. ${ }^{2}$ Rod-like colloidal semiconductor nanocrystals such as CdSe quantum rods or CdSe/CdS dot-in-rods on the other hand more strongly absorb and emit light polarized parallel to their long axis.,

\footnotetext{
${ }^{a}$ Electronics and Information Systems Department, Ghent University, Zwijnaarde 15, B-9052 Gent, Belgium. E-mail: mohammad.mohammadimasoudi@elis.ugent.be; kneyts@elis.ugent.be

${ }^{b}$ Physics and Chemistry of Nanostructures, Ghent University, Krijgslaan 281-S3, 9000 Gent, Belgium

${ }^{c}$ Center for Nano- and Biophotonics, Ghent University, Belgium

$\dagger$ Electronic supplementary information (ESI) available: Synthesis of CdSe/CdS nanorods; photograph and transmission measurement of CdSe/CdS NR suspension in dodecane; characteristic information and obtained data on CdSe/CdS NRs; rotational relaxation frequency; polarization ratio. See DOI: $10.1039 / \mathrm{c} 6 \mathrm{ra02620f}$
}

Although use can be made of the anisotropic properties of single nanorods, ${ }^{2,7,8}$ applications such as polarized light emitting diodes, ${ }^{9}$ photovoltaic energy conversion, optical sensors or switches ${ }^{\mathbf{1 0 - 1 3}}$ require layers or volumes with large ensembles of aligned nanorods. In this context, various methods for the collective alignment of colloidal nanorods have been explored. Focusing on colloidal quantum rods, these either exploit the tendency of nanorods to self-assemble by aligning their long axes upon drying or dispersion destabilization or involve the use of external forces to impose rod alignment. Depending on the actual conditions, slow solvent evaporation on a solid substrate or a liquid subphase results in nanorod films aligned with their long axis parallel or perpendicular to the substrates. ${ }^{\mathbf{1 4 - 1 9}}$ Alternatively, alignment by external forces has been demonstrated using mechanical rubbing of a predeposited film of randomly oriented quantum $\operatorname{rods}^{20}$ or by means of electric fields applied either during solvent drying ${ }^{21-23}$ or to achieve electrophoretic deposition. ${ }^{24}$

Besides the alignment of nanorods during deposition, a number of studies have addressed the alignment of quantum rods in solution. Various reports describe the organization of nanorods in two-dimensional sheets of a single layer of parallel quantum rods by depletion attraction, ${ }^{25}$ specific surface functionalization schemes ${ }^{\mathbf{2 6}}$ or a reduction of the dispersion stability. ${ }^{27,28}$ Although it proved possible to orient such 2D sheets in solution by magnetic fields, ${ }^{29}$ the size of the sheets leads to considerable scattering of light and results in patchy deposits upon solvent drying. Opposite from this alignment-byaggregation, the collective orientation or alignment of nonaggregated nanorods is possible by means of an electric field. 
Although electrical alignment has mainly been used to characterize nanorod properties such as the dipole moment ${ }^{30}$ or the absorption anisotropy, ${ }^{31}$ it can be regarded as a technical solution to produce functional solid films with strong anisotropy in optical absorption, emission or scattering. For example, alignment-in-solution can be achieved in a polymerizable medium, where a triggered polymerization can lock the rod orientation to produce self-supporting films or sheets of aligned nanorods. Such an approach can have several advantages. Depending on the electrode configuration, the alignment can be homogenous or follow a pattern. Moreover, in-solution alignment by an electric field is also possible at low nanorod concentrations, thus leading to films with a desired absorption coefficient for, e.g., optical down-conversion applications in lighting, displays or luminescent solar concentrators.

Despite the potential of in-solution alignment of nanorods by electric fields, both theoretical and experimental aspects have only been partially studied in the literature. Ruda et al. gave a theoretical description of the alignment of gold nanorods based on the anisotropy of the induced dipole moment..$^{32}$ In the case of semiconductor nanorods on the other hand, the alignment by alternating electric fields is typically attributed to a permanent dipole along their long axis, ${ }^{21,30,31}$ although the presence of a permanent dipole could not be confirmed by electrostatic force microscopy. ${ }^{33}$ In addition, there are no reports about reaching the regime of full nanorod alignment in the literature. Using the available combination of applied fields and quantum rod dipoles, Kamal et al. only obtained partial orientation, as the electrostatic energy gained by orienting the rods was at best about equal to the thermal energy, ${ }^{31}$ while an even weaker orientation can be deduced from the figures provided by Li et $a .^{30}$

In this paper, we demonstrate that colloidal CdSe/CdS nanorods that are dispersed in a non-polar solvent can be quasi fully aligned by applying sufficiently strong alternating electric fields. The degree of alignment is monitored by measuring the transmission ${ }^{31}$ as a function of time during the application of a time-dependent voltage. The measurement results are interpreted by using a dynamic model that encompasses the viscous drag of the solvent, the rotational diffusion of the nanorods, the torque due to the permanent dipole moment and the (induced) dielectric torque. The transition from random to full alignment as a function of the electric field enables us to attribute the alignment to the nanorods' permanent dipole and determine the dipole's magnitude. In addition, we show that full alignment and relaxation are established with characteristic time constants of $50 \mu \mathrm{s}$ or less. Finally, we demonstrate that the regime of full alignment is ideal to quantify the absorption anisotropy of colloidal NRs, where we find that the small dielectric mismatch between the CdS rod and the CdSe dot makes that CdSe-dot and CdS-rod related optical transitions feature a similar absorption anisotropy.

\section{Experimental}

\section{CdSe/CdS nanorod synthesis}

CdSe/CdS dot-in-rods are synthesized according to a procedure described in the literature (see ESI S1 $\uparrow$ for synthesis details). ${ }^{23}$ In (a)
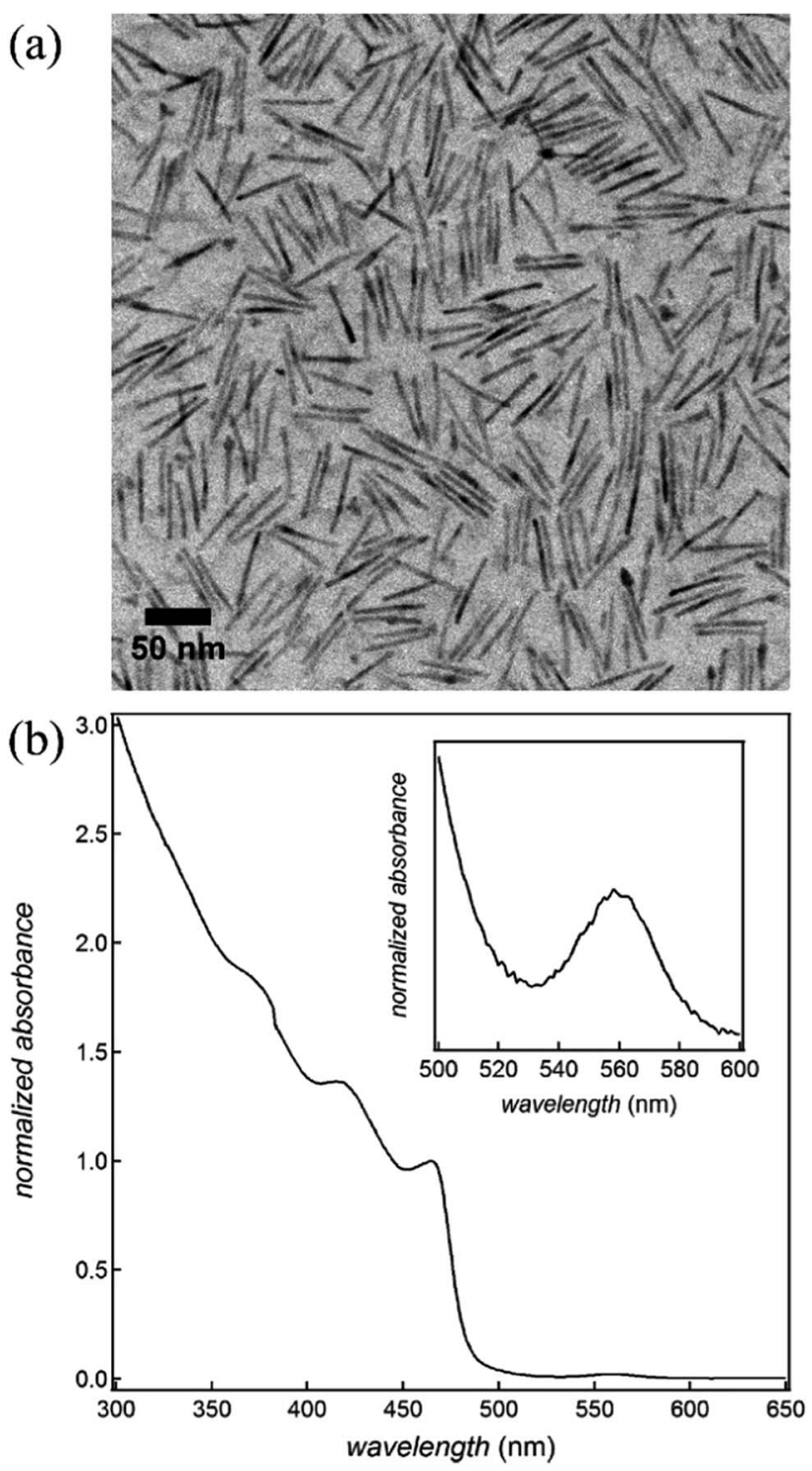

Fig. 1 (a) TEM micrograph of the CdSe/CdS nanorods (b) absorption spectrum of the CdSe/CdS nano rod dispersion, normalized at the first CdS absorption feature at $\approx 470 \mathrm{~nm}$ (inset: magnification on the range 500 to $600 \mathrm{~nm}$ highlighting the absorption feature of the CdSe cores).

brief, CdSe quantum dots (QDs) with a wurtzite structure and an average diameter of $2.3 \mathrm{~nm}$ are synthesized first. On these core QDs, an anisotropic CdS shell is grown using phosphonic acids as the ligands to obtain CdSe/CdS dot-in-rods, which we will denote as CdSe/CdS nanorods (NRs). Fig. 1 provides an overview of the most important characteristics of the NRs used in this study. Based on the transmission electron micrograph (Fig. 1a), their average diameter and length are estimated at 4.8 and $51.5 \mathrm{~nm}$, respectively. Moreover, their absorbance spectrum (Fig. 1b) indicates that they have a first exciton or band-gap transition at $560 \mathrm{~nm}$, whereas the absorbance by the CdS rod starts dominating at wavelengths below $500 \mathrm{~nm}$. 

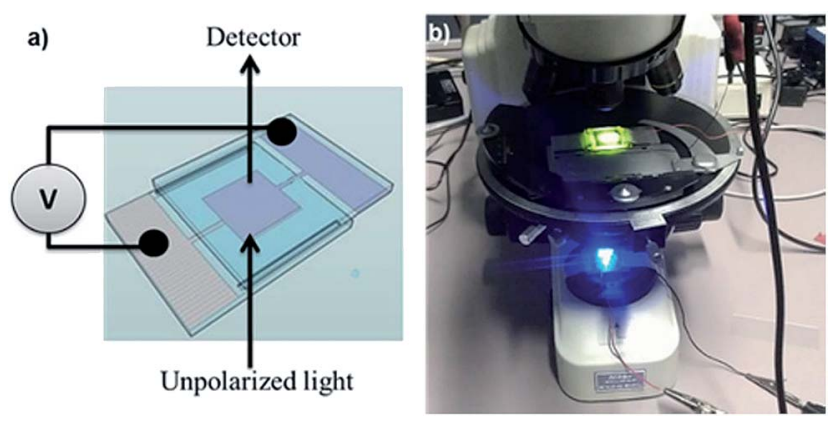

Fig. 2 (a) Schematic view of the cell used to align dispersed nanorods by means of electric fields and (b) image of the microscope transmission setup.

\section{Quantum rod alignment by electric fields}

As shown in Fig. 2a, a cell is made by joining two glass substrates with $1 \mathrm{~cm}^{2}$ ITO electrodes with glue containing 20 or $50 \mu \mathrm{m}$ spacer beads (Sekisui Chemicals) to ensure a uniform cell gap $d$ between the electrodes.

The cell is filled with the CdSe/CdS NR suspension by capillary force (see ESI S2 $\uparrow$ ). A function generator (TTi-TG315) and a voltage amplifier are used to apply a high AC voltage $V$ with a frequency between $50 \mathrm{~Hz}$ and $40 \mathrm{kHz}$ over the cell. This results in electric fields - estimated as $\boldsymbol{E}=V / d$ - of up to $40 \mathrm{~V}$ $\mu \mathrm{m}^{-1}$ in the dispersion. The transmission of blue $(470 \mathrm{~nm})$ and green $(560 \mathrm{~nm})$ LED light is measured using a photodetector (FLC electronics-PIN20) mounted on a microscope (Nikoneclipse E400). Fig. $2 \mathrm{~b}$ shows part of the setup including the cell and the blue LED.

\section{Theoretical background}

\section{Orientation of spheroids in an electric field}

As sketched in Fig. 3a, the application of a voltage difference across the cell, and thus an electric field inside the dispersion, will change the random orientation of the long axis of the NRs into a preferential alignment along the electric field. To describe this electric-field driven alignment of NRs dispersed in an apolar liquid, we consider both their permanent and induced (dielectric) dipole moments. Whereas CdSe/CdS NRs have a permanent dipole moment that is related to their noncentro-symmetric wurtzite crystal structure, the induced dipole moment cannot be neglected a priori since semiconductor NRs typically have a considerably higher dielectric constant than the surrounding apolar liquid.

To assess the characteristic times determining the motion of NRs under the action of an alternating field, we use a dynamic equation that takes into account both the dielectric and dipolar torques, thermal fluctuations and rotational viscosity of the NRs. When a NR - approximated as a prolate ellipsoid of revolution is placed in a host medium with dielectric constant $\varepsilon_{\mathrm{h}}$ in which a homogeneous external field $\boldsymbol{E}$ is present, the induced dipole moment $\boldsymbol{p}_{\text {ind }}$ with respect to the host medium amounts to:

$$
\boldsymbol{p}_{\text {ind }}=\alpha_{\|} \varepsilon_{0} \boldsymbol{E}_{\|} \mathbf{1}_{\|}+\alpha_{\perp} \varepsilon_{0} \boldsymbol{E}_{\perp} \mathbf{1}_{\perp}
$$

here, the unit vectors $\mathbf{1}_{\|}$and $\mathbf{1}_{\perp}$ are mutually orthogonal, pointing along and perpendicularly to the long axis of the ellipsoid, respectively (see Fig. 3c). The field components $\boldsymbol{E}_{\|}$and $\boldsymbol{E}_{\perp}$ are the respective projections of the electric field on $\mathbf{1}_{\|}$and $\mathbf{1}_{\perp}$. For an anisotropic ellipsoid of revolution with dielectric constants $\varepsilon_{\|}$and $\varepsilon_{\perp}$, the concomitant polarizabilities $\alpha_{\|}$and $\alpha_{\perp}$ are given by: ${ }^{34}$

$$
\begin{aligned}
\alpha_{\|} & =\frac{\varepsilon_{\|}-\varepsilon_{\mathrm{h}}}{\varepsilon_{\mathrm{h}}+L_{\|}\left(\varepsilon_{\|}-\varepsilon_{\mathrm{h}}\right)} V \\
\alpha_{\perp} & =\frac{\varepsilon_{\perp}-\varepsilon_{\mathrm{h}}}{\varepsilon_{\mathrm{h}}+L_{\perp}\left(\varepsilon_{\perp}-\varepsilon_{\mathrm{h}}\right)} V
\end{aligned}
$$

In the above expression, $V$ represents the volume of a NR and $L_{\|}$and $L_{\perp}$ are the depolarization factors for fields parallel and perpendicular to the NR long axis, respectively. With $a$ and $b$ the length of the corresponding semi-axes of the ellipsoid (see Fig. 3c), these read: ${ }^{35}$

$$
\begin{aligned}
& L_{\|}=\frac{b^{2}}{a^{2}-b^{2}}\left[\frac{a}{2 \sqrt{a^{2}-b^{2}}} \ln \frac{a+\sqrt{a^{2}-b^{2}}}{a-\sqrt{a^{2}-b^{2}}}-1\right] \\
& L_{\perp}=\frac{1-L_{\|}}{2}
\end{aligned}
$$

The induced dipole moment leads to a dielectric or induced torque on the NR given by:

$$
\boldsymbol{T}_{\text {ind }}=\boldsymbol{p}_{\text {ind }} \times \boldsymbol{E}=\frac{1}{2}\left(\alpha_{\|}-\alpha_{\perp}\right) \sin 2 \theta \varepsilon_{0} \boldsymbol{E}^{2} \mathbf{1}_{\perp}^{\prime}
$$

here, $\theta$ is the angle between the long axis of the ellipsoid and the electric field, whereas $\mathbf{1}^{\prime}{ }_{\perp}$ is the unit vector perpendicular to both $\mathbf{1}_{\|}$and $\mathbf{1}_{\perp}$ (see Fig. $3 \mathrm{c}$ ).

If the NR has a permanent dipole moment $\boldsymbol{p}$ along the long axis of the NR, then the external electric field leads to an additional dipolar torque $\boldsymbol{T}_{\mathrm{dip}}$ :

$$
T_{\text {dip }}=p \times E=p E \cos \theta \mathbf{1}_{\perp}^{\prime}
$$

Including the two torques into the dynamic equation of reorientation yields the following differential equation for the orientation angle $\theta$ :

$$
\begin{aligned}
I \frac{\mathrm{d}^{2} \theta}{\mathrm{d} t^{2}}+\gamma_{\mathrm{r}} \frac{\mathrm{d} \theta}{\mathrm{d} t}= & \sqrt{k_{\mathrm{B}} T \gamma_{\mathrm{r}}} \delta(t)+\boldsymbol{p} \boldsymbol{E} \cos \theta \\
& +\frac{1}{2}\left(\alpha_{\|}-\alpha_{\perp}\right) \sin 2 \theta \varepsilon_{0} \boldsymbol{E}^{2} \mathbf{1}_{\perp}^{\prime}
\end{aligned}
$$

The first term of eqn (6) contains the moment of inertia $I$ and is usually negligible for small ellipsoids, except at very high frequencies (i.e., $\mathrm{MHz}$ range). The second term represents the viscous torque, whereas the first term on the right is the torque due to Brownian motion, ${ }^{36}$ with $k_{\mathrm{B}}$ the Boltzmann constant and $T$ the absolute temperature. In both expressions, $\gamma_{\mathrm{r}}$ is the rotational viscosity: ${ }^{32}$

$$
\gamma_{\mathrm{r}}=\frac{16 \pi \eta \sqrt{a^{2}-b^{2}}\left(a^{4}-b^{4}\right)}{3\left(\frac{a^{2}-b^{2}}{2}\right) \ln \frac{a+\sqrt{a^{2}-b^{2}}}{a-\sqrt{a^{2}-b^{2}}}-a \sqrt{a^{2}-b^{2}}}
$$



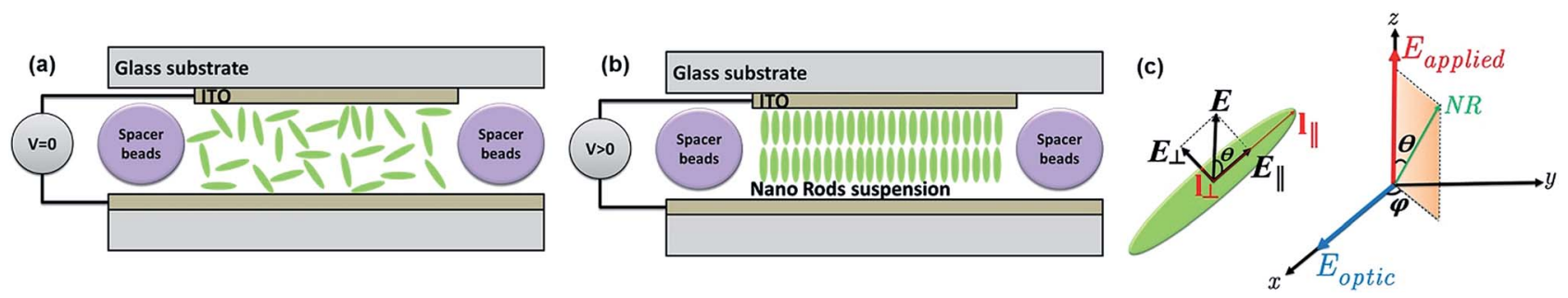

Fig. 3 Orientation of NRs in suspension in dodecane (a) without and (b) with applied voltage. (c) The electric field is perpendicular to the ITO electrodes and $\theta$ is the angle between the applied electric field and the NR long axis. The components of the applied electric field parallel and perpendicular to the long axis $l_{\|}$are called $E_{\|}$and $E_{\perp}$ respectively.

here, $\eta$ is the dynamic viscosity of the medium. In the case of NRs stabilized by organic ligands, $a$ and $b$ should be seen as the semi-axes of the NR, augmented with the length of the ligands $(2 \mathrm{~nm})$.

Using eqn (6), we can estimate the typical time $\tau_{\text {on }}$ it takes to align an ellipsoid when switching on a sufficiently strong electric field by neglecting the Brownian term, replacing the derivative by the ratio $\Delta \theta / \tau_{\mathrm{on}}$, and setting $\Delta \theta=\pi / 4$ :

$$
\begin{aligned}
\tau_{\mathrm{on}} & =\frac{\pi \gamma_{\mathrm{r}}}{2 \sqrt{2} \boldsymbol{p} \boldsymbol{E}+2\left(\alpha_{\|}-\alpha_{\perp}\right) \varepsilon_{0} \boldsymbol{E}^{2}} \\
f_{\mathrm{on}} & =\frac{1}{2 \pi \tau_{\mathrm{on}}}
\end{aligned}
$$

The typical time for reorientation when switching off the voltage is called the relaxation time $\tau_{\text {rel }}$, which is usually longer than $\tau_{\text {on }}$. This time and the relaxation frequency can be estimated using the Debye-Perrin model and eqn (6):

$$
\begin{gathered}
\tau_{\text {rel }}=\frac{\gamma_{\mathrm{r}}}{k_{\mathrm{B}} T} \\
f_{\text {rel }}=\frac{k_{\mathrm{B}} T}{2 \pi \gamma_{\mathrm{r}}}
\end{gathered}
$$

The excess electrostatic energy $U_{\mathrm{el}}$ of a stationary ellipsoid in an electric field is given by:

$$
U_{\mathrm{el}}=-\boldsymbol{p} \boldsymbol{E} \cos \theta-\frac{1}{2}\left[\alpha_{\perp}+\left(\alpha_{\|}-\alpha_{\perp}\right) \cos ^{2} \theta\right] \varepsilon_{0} \boldsymbol{E}^{2}
$$

In the quasi-static case, when the frequency of the field is well below $f_{\text {rel }}$ and $f_{\text {on }}$, the probability $P(\theta)$ to find an ellipsoid within a given solid angle making an angle $\theta$ with the long axis of the rod is proportional to the Boltzmann distribution:

$$
P(\theta) \propto \exp \left(-\frac{U_{\mathrm{el}}}{k_{\mathrm{B}} T}\right)
$$

\section{Light absorbance by dispersed ellipsoidal particles}

When a light absorbing ellipsoidal nano-object is dispersed in a non-absorbing solvent, the effective absorption coefficient for light depends on the orientation of the particle relative to the incident electric field. Based on the depolarization factors parallel and perpendicular to the long axis of the ellipsoid, we can write down the intrinsic absorption coefficients based on the Maxwell Garnett model ${ }^{37}$

$$
\begin{aligned}
& \mu_{\|}=\frac{2 \pi \varepsilon_{\|, \mathrm{I}}}{\lambda n_{\mathrm{h}}}\left|\frac{\varepsilon_{\mathrm{h}}}{\varepsilon_{\mathrm{h}}+L_{\|}\left(\varepsilon_{\|, \mathrm{R}}+\mathrm{i} \varepsilon_{\|, \mathrm{I}}-\varepsilon_{\mathrm{h}}\right)}\right|^{2} \\
& \mu_{\perp}=\frac{2 \pi \varepsilon_{\perp, \mathrm{I}}}{\lambda n_{\mathrm{h}}}\left|\frac{\varepsilon_{\mathrm{h}}}{\varepsilon_{\mathrm{h}}+L_{\perp}\left(\varepsilon_{\perp, \mathrm{R}}+\mathrm{i} \varepsilon_{\perp, \mathrm{I}}-\varepsilon_{\mathrm{h}}\right)}\right|^{2}
\end{aligned}
$$

In this expression, $n_{\mathrm{h}}$ is the refractive index of the host solvent and $\varepsilon_{\|, \mathrm{R}}+\mathrm{i} \varepsilon_{\|, \mathrm{I}}$ and $\varepsilon_{\perp, \mathrm{R}}+\mathrm{i} \varepsilon_{\perp, \mathrm{I}}$ are the complex dielectric constant of the nanorod for the considered wavelength in the directions parallel and perpendicular to the NR long axis, respectively. In the typical situation for semiconductor NRs in an apolar solvent, their magnitude exceeds $\varepsilon_{\mathrm{h}}$, resulting in a screening of the electric field by the NRs. For a prolate ellipsoid, $L_{\|}$is smaller than $L_{\perp}$ such that the absorption coefficient for parallel orientation is the larger of the two. For an ellipsoid with inclination $\theta$ and azimuth $\varphi$ as shown in Fig. 3c, the absorption coefficient will be an average of $\mu_{\|}$and $\mu_{\perp}$, weighted by the square of the projection of the electric field:

$$
\mu(\theta, \varphi)=\mu_{\|} \cos ^{2} \varphi \sin ^{2} \theta+\mu_{\perp}\left(1-\cos ^{2} \varphi \sin ^{2} \theta\right)
$$

For an ensemble of ellipsoids, the absorption coefficient $\mu$ in eqn (13) is obtained by averaging over all orientations of the NR long axis, weighted with the corresponding Boltzmann factor (eqn (11)):

$$
\mu=\frac{\int_{0}^{2 \pi} \int_{0}^{\pi} P(\theta) \mu(\theta, \varphi) \sin \theta \mathrm{d} \theta \mathrm{d} \varphi}{\int_{0}^{2 \pi} \int_{0}^{\pi} P(\theta) \sin \theta \mathrm{d} \theta \mathrm{d} \varphi}
$$

Finally, the absorbance $A$ of a dispersion of ellipsoidal nanoobjects is given by the products of the intrinsic absorption coefficient $\mu$ and the volume fraction $f$ of the ellipsoids and the path length $d$ of light through the dispersion, divided by the natural logarithm of 10 :

$$
A=\mu \frac{f d}{\ln 10}
$$




\section{Results and discussion}

Full alignment of colloidal NRs by electric fields

To explore the alignment of dispersed colloidal NRs by electric fields, a dodecane-based dispersion of CdSe/CdS NRs is loaded in a cell as described in the Experimental section. As a fraction of the NRs will carry an electric charge, ${ }^{38}$ application of a DC field will make the NRs drift to the electrodes. To avoid artefacts due to NR accumulation at the electrodes, we use AC fields with a period shorter than the cell transit time $\tau_{\text {cell }}=d / u \boldsymbol{E}$ where $u$ is the mobility of the NRs. As shown in the ESI, $\dagger$ NRs in a $50 \mu \mathrm{m}$ cell have a cell transit time of $\approx 5 \mathrm{~ms}$ for fields of $17 \mathrm{~V} \mu \mathrm{m}^{-1}$. Therefore, we use frequencies higher than $200 \mathrm{~Hz}$ to study NR alignment.

A first measurement involves the optical transmission of a 50 $\mu \mathrm{m}$ cell filled with a $1 \mu \mathrm{M}$ dispersion of NRs in dodecane at 470 $\mathrm{nm}$ in the presence of a sinusoidal AC electric field. An example is given in Fig. 4a, where it can be seen that the two extreme values of the electric field during a single period both correspond to a maximum increase of the transmission (see also the transmission of the cell in presence of a block wave electric field in ESI, Fig. S3†). From these transmission measurements, the absorbance $A$ by the NR dispersion is calculated as:

$$
A=-\log \frac{I_{\mathrm{T}}}{I_{0}}
$$

here, $I_{0}$ is the light intensity passing through the cell loaded with dodecane only, while $I_{\mathrm{T}}$ is the (possibly time dependent) intensity transmitted through the NR-loaded cell. Note that scattering by the nanorods is neglected here. One readily sees that the increased transmission upon application of an electric field corresponds to a drop of the absorbance. This is in agreement with NRs aligning their long axes parallel to the AC electric field and perpendicular to the electric field of the incident light, where the absorbance drop is due to the higher depolarization factor for fields along the short axis of the CdSe/ CdS NRs $\left(L_{\perp}=0.49\right.$ instead of $L_{\|}=0.018$, see ESI S4, Table $\mathrm{S} 1 \dagger)$.

In order to eliminate the trivial influence of cell thickness and NR concentration from the measurements, we introduce the relative change of the absorbance $\Delta A / A_{0}$ (usually $<0$ ):

$$
\frac{\Delta A}{A_{0}}=\frac{A-A_{0}}{A_{0}}
$$

here, $A_{0}$ is the absorbance of the NR-loaded cell in the absence of an electric field. Fig. $4 \mathrm{~b}$ represents the variation of the minimum of $\Delta A / A_{0}$ as a function of the applied electric field. One sees that whereas increasing the electric field initially leads to a more pronounced drop of the absorbance, $\Delta A / A_{0}$ levels off

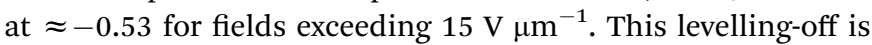
also apparent from the variation of $\Delta A / A_{0}$ over a single period of the AC field. When applying for example a $1 \mathrm{kHz}$ field with

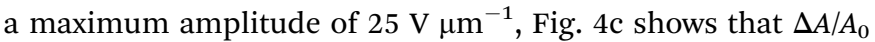
hits a plateau each time the field is around its extreme values. The observations in Fig. $4 \mathrm{~b}$ and $\mathrm{c}$ are in good agreement with the simulated data for the alignment of nanorods with a permanent dipole moment in a homogeneous electric field.
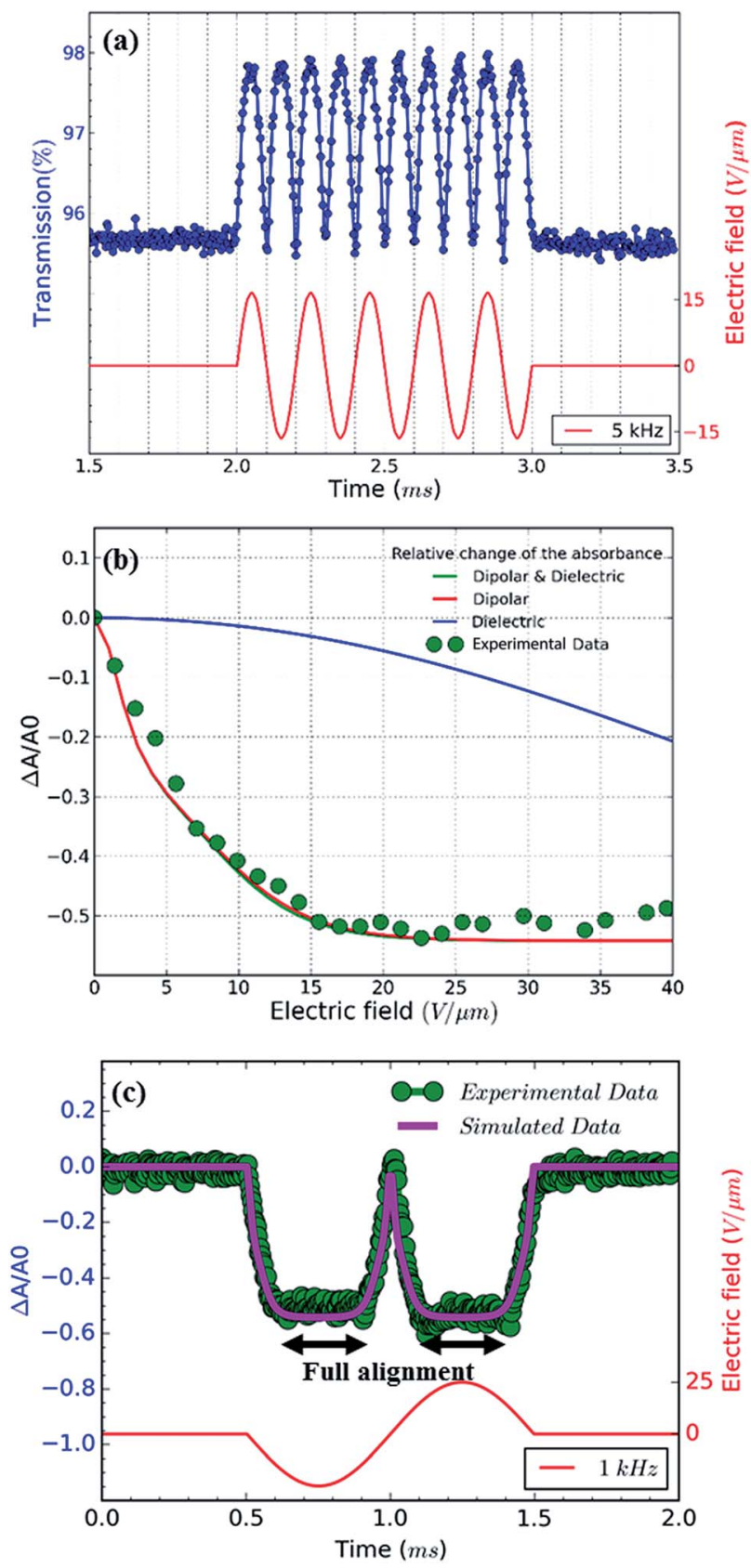

Fig. 4 (a) Transmission measurements (blue dots) of a CdSe/CdS NR suspension for blue light $(\sim 470 \mathrm{~nm}$ ) with and without the presence of a sinusoidal $A C$ electric field with a frequency of $5 \mathrm{kHz}$ and an amplitude of $17 \mathrm{~V} \mu \mathrm{m}^{-1}$ (red line). (b) Minimum value of the relative change of the absorbance of the $1 \mu \mathrm{MNR}$ dispersion in the presence of an $\mathrm{AC}$ electric field with a frequency of $1 \mathrm{kHz}$ as a function of peak value of the electric field. Measurements (green dots) and simulations assuming only dielectric torque (blue), only dipolar torque (red) and both dielectric and dipolar torques (green, almost identical to red). (c) Relative change of the measured (green dots) and simulated (magenta) absorbance of the $1 \mu \mathrm{M}$ NR dispersion for blue $(\sim 470 \mathrm{~nm})$ light in the presence of an AC electric field with frequency $1 \mathrm{kHz}$ and amplitude 25 $\vee \mu \mathrm{m}^{-1}$ (red).

For high voltages, the theoretical model predicts that the root mean square of the inclination angle $\theta$ becomes, within a few degrees, equal to $0^{\circ}$ or $180^{\circ}$. We call this full alignment. The 
simulations means that the average deviation angle from perfect alignment is around 9 degrees for the highest voltage applied. Fig. 4c also suggests that at sufficiently high amplitudes, the NRs will be fully aligned during most of the time, except for small time intervals in which the field changes sign. Clearly, such a condition is highly beneficial if the rod orientation is to be locked in by, for example, a triggered polymerization of the surrounding medium.

\section{Dynamical properties of colloidal NR alignment}

Next to the levelling off of the absorbance change, Fig. 4c also makes clear that the change in absorbance is established without noticeable delay. This indicates that at the fields and frequencies used, the NRs readily flip from a $\theta=0^{\circ}$ to a $\theta=180^{\circ}$ orientation when the field changes direction (see Fig. $3 \mathrm{c}$ for a definition of the angle $\theta$ ).

Hence, the NR reorientation occurs on a time scale much shorter than the $1 \mathrm{~ms}$ period of the AC field, an outcome in line with the dynamical properties of NR (re)orientation. Indeed, using eqn (7) and (9), the rotational relaxation frequency $f_{\text {rel }}$ in dodecane of the CdSe/CdS NRs used here can be estimated at 13 $\mathrm{kHz}$ (see ESI S4, Table S1†).

Hence, fields with a frequency well below $13 \mathrm{kHz}$, should give the NRs enough time to establish a quasi-equilibrium Boltzmann distribution that follows the variation of the electric field, thus bringing them from random orientation to full alignment. In line with this, increasing the AC frequency from 1 to $10 \mathrm{kHz}$ only changes the frequency at which the transmission changes, without affecting the limiting value corresponding to full alignment (see ESI S5 $\dagger$ ). Hence, the only difference between a 1 and a $10 \mathrm{kHz}$ field is that the latter makes the NRs flip faster between the $\theta=0^{\circ}$ to a $\theta=180^{\circ}$ orientation.

At frequencies above $f_{\text {rel }}$, we expect that the distribution will not return to random alignment and the minimum in the transmission will not be as low as the zero-field transmission. Moreover, the NRs will stop reacting to the AC field once the frequency exceeds the turn on frequency $f_{\text {on }}$, which is estimated at $\approx 100 \mathrm{kHz}$ in this case (see ESI S4, Table S1†). This description of the NR orientation dynamics is further confirmed by the response of the transmission to a single block pulse with an amplitude of $17 \mathrm{~V}^{-1}$. As shown in Fig. 5, the regimes of full alignment and de-alignment are reached within less than $50 \mu \mathrm{s}$.

\section{Permanent versus induced dipole moments}

As the absorbance of the CdSe/CdS NRs at $470 \mathrm{~nm}$ will be dominated by the CdS rod (Fig. 1b), the bulk optical constants of CdS can be used to estimate the dielectric function of the nanorod in the expressions for the polarizability and the absorption coefficient of dielectric ellipsoids, i.e., eqn (2) and (14), respectively. The thus calculated polarizability and absorption coefficients at $470 \mathrm{~nm}$ have been summarized in ESI S4, Table S1.† Using these figures, the degree of orientation and the concomitant relative absorbance change can be calculated if only the dielectric torque were present. The thus predicted dependence of $\Delta A / A_{0}$ on the electric field strength has been added to Fig. $4 \mathrm{~b}$, where it follows that fields of more than

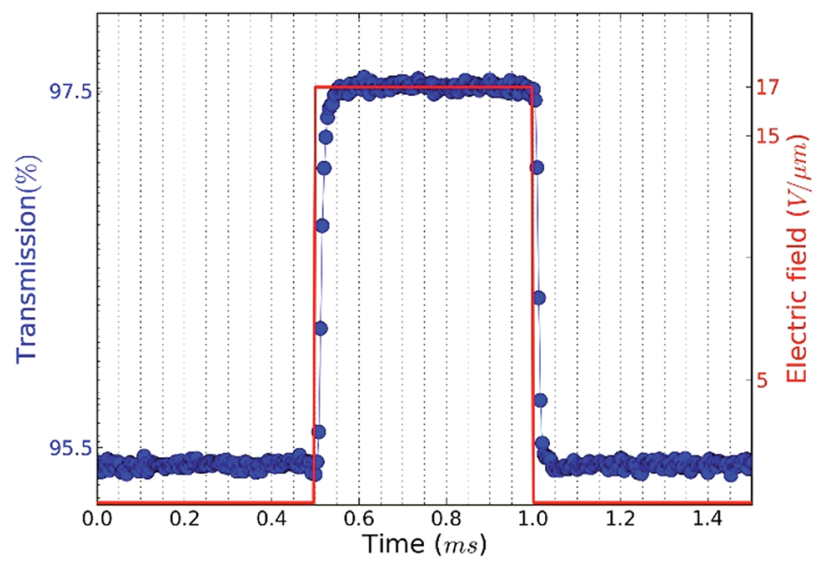

Fig. 5 Transmission versus time of a CdSe/CdS NR suspension in dodecane when a pulse with amplitude of $17 \mathrm{~V} \mu \mathrm{m}^{-1}$ is applied.

$40 \mathrm{~V} \mu \mathrm{m}^{-1}$ would be needed to obtain an appreciable alignment of the NRs. We therefore conclude that the (full) alignment of the NRs is indeed due to the permanent dipole moment of the NRs. Considering the NR dipole moment $\boldsymbol{p}$ as an adjustable parameter, the field dependence of $\Delta A / A_{0}$ can again be predicted. Fig. $4 \mathrm{~b}$ shows that taking $\boldsymbol{p}=1500 \mathrm{D}$, a typical value for the CdSe/CdS NRs used here, makes the simulated field dependency of $\Delta A / A_{0}$ closely match the experimental data. We stress that this predicted trend depends on a single adjustable parameter, i.e., the dipole moment $\boldsymbol{p}$, which mainly determines the region of electric fields where the NRs change from random to full alignment. The high-field limit of $\Delta A / A_{0}$ on the other hand only depends on the difference in absorption coefficient of fully aligned $\left(\mu_{\perp}\right)$ and randomly aligned $\left(\mu_{0}\right)$ quantum rods:

$$
\lim _{E \rightarrow \infty} \frac{\Delta A}{A_{0}}=\frac{\mu_{\perp}-\mu_{0}}{\mu_{0}}
$$

Inserting the estimated absorption coefficients (see ESI, Table $1 \dagger$ ), a limit of -0.54 is obtained, in line with the experimental findings.

\section{Absorption anisotropy at the band-edge transition}

As indicated by eqn (18), the regime of full alignment provides a way to directly determine the absorption anisotropy of colloidal NRs at any given wavelength. We therefore extended the analysis to the NR absorbance at the CdSe/CdS first exciton transition at around $560 \mathrm{~nm}$ (see Fig. 1b), which is due to electronic transitions between quantized states in the CdSe core. To adjust for the absorbance difference at 470 and $560 \mathrm{~nm}$ of the CdSe/CdS NRs, we had to analyze NR dispersions with a concentration 10 times higher than used for the previous measurements. Hence, we now measure the transmission of green light $(\approx 560 \mathrm{~nm}$ ) through a $50 \mu \mathrm{m}$ thick cell filled with a $10 \mu \mathrm{M}$ NR dispersions in the presence of an AC electric field $\left(17 \mathrm{~V} \mu \mathrm{m}^{-1}, 1 \mathrm{kHz}\right)$. Fig. 6a shows that the AC field leads to a similarly oscillating increased transmission - meaning a decrease in absorbance - as observed before at $470 \mathrm{~nm}$. 
Hence, also the CdSe core transitions exhibit a marked absorption anisotropy. An issue with the more concentrated dispersions used here is that a considerable fraction of the NRs form aggregates that are not oriented by the applied electric field. Fig. $6 \mathrm{~b}$ therefore compares the relative change of the absorbance determined at $560 \mathrm{~nm}$ with that determined at 470 $\mathrm{nm}$ for the same dispersion under the same conditions of the applied field. Since this transmission change would reach -0.54 if all rods aligned, the actual plateau value can be used to estimate the fraction of NRs that can be aligned and thus calibrate the transmission changes at $560 \mathrm{~nm}$.

Fig. $6 \mathrm{~b}$ shows that this calibration is actually quite straightforward since the high-field limit of $\Delta A / A_{0}$ (eqn (17)) at $560 \mathrm{~nm}$ due to CdSe core absorption is very similar to the one measured at $470 \mathrm{~nm}$, which is due to absorption in the CdS rod. Hence, a quite similar corrected limiting value of $\Delta A / A_{0}$ in the range -0.5 to -0.55 is obtained at the band-gap transition as well. This result may seem surprising since the core is a spherically symmetric object, yet it can be understood by realizing that the dielectric mismatch between the CdSe core and the CdS shell is small.
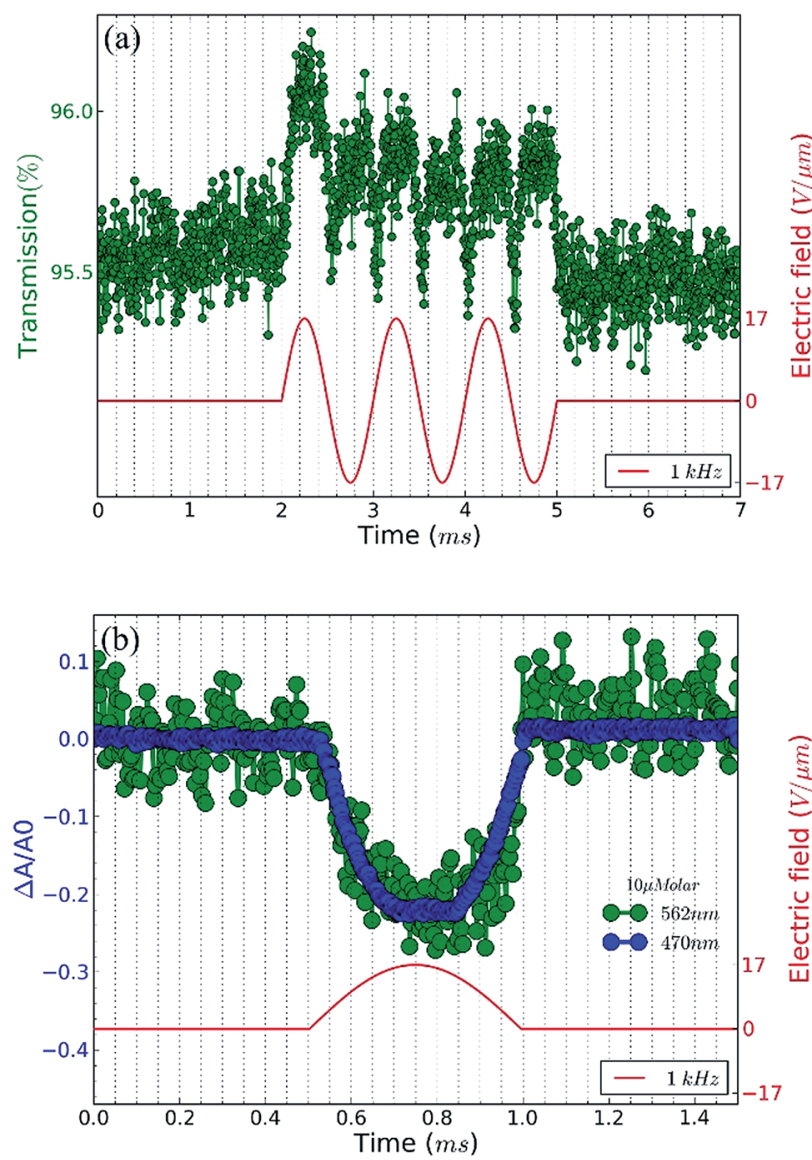

Fig. 6 (a) Transmission measurement (green dots) of a NR suspension for green ( $\sim 560 \mathrm{~nm}$ ) light in the presence of an AC electric field with frequency $1 \mathrm{kHz}$ and amplitude $17 \mathrm{~V} \mu \mathrm{m}^{-1}$ (red) (b) Relative change of the measured absorbance of the $10 \mu \mathrm{M}$ NR dispersion for blue light (blue dots) and green light (green dots) in the presence of an AC electric field with frequency $1 \mathrm{kHz}$ and amplitude $17 \mathrm{~V} \mu \mathrm{m}^{-1}$ (red).
To clarify this point, Fig. 7 shows the electric field distribution within a CdS ellipsoid encompassing a CdSe sphere and surrounded by dodecane as a host. This field has been obtained from a 3D finite element field calculation modelling all materials by means of the real parts of the anisotropic bulk dielectric functions at $560 \mathrm{~nm}$ (see ESI S4, Table S2†). Regardless of the orientation of the NR relative to the external field, we find that the internal field in the CdSe core amounts to $\approx 95 \%$ of the field in the CdS host.

Moreover, the electric field in the rod (and thus the sphere) is almost identical to the external electric field when the applied field is parallel to the $c$-axis of the rod, while it is only about half the applied external field for the perpendicular orientation.

These internal fields closely agree with the internal fields in CdS-only ellipsoids with depolarization factors $L_{\|}=0.018$ and $L_{\perp}=0.49$ dispersed in dodecane. The CdSe core thus experiences the same enhanced or reduced screening of the internal field as the CdS rod for incident optical fields perpendicular to or parallel with the long axis of the NR. Hence the largely similar absorption anisotropy for the quantum-dot band gap transition.

Note that the experimentally determined high-field limit of $\Delta A / A_{0}$ corresponds to a polarization ratio of $64 \%$ for the emission (see ESI, S6†), which is similar to the experimentally determined values of up to $75 \%$ as determined on single CdSe/ CdS NRs. ${ }^{5}$

\section{Conditions to reach full alignment}

According to formula (11), full alignment is obtained when the difference in electrostatic energy $U_{\mathrm{el}}$ between parallel and perpendicular alignment is considerably larger than $k T$.

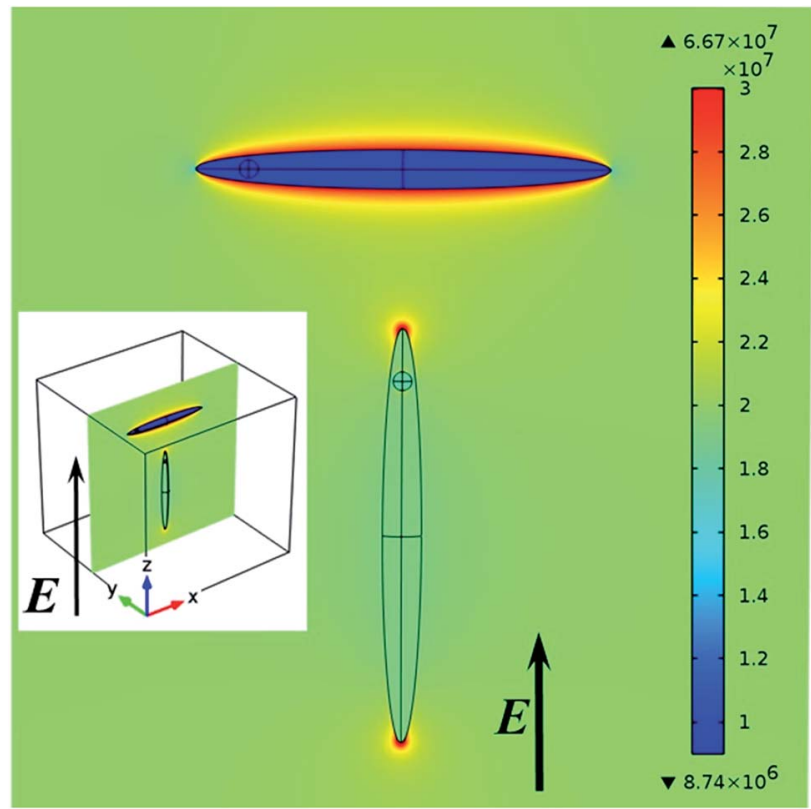

Fig. 7 Simulation of the field distribution $\left(\mathrm{V} \mathrm{m}^{-1}\right)$ inside a dot in rod using the bulk dielectric function for incident light parallel and perpendicular to the $c$-axis of the rod at $560 \mathrm{~nm}$. The minimum and maximum values of the field are indicated next to the triangles (inset: 3D structure shows the position of the NRs in the medium). 
Because we found that the contribution from the induced dipole moment is relatively small, the difference in electrostatic energy is in good approximation given by $-\boldsymbol{E} \boldsymbol{p}$ according to eqn (10). Based on the experimental result that a dipole moment of $1500 \mathrm{D}$ requires a threshold field $\boldsymbol{E}_{\text {th }}$ of $17 \mathrm{~V} \mu \mathrm{m}^{-1}$, we can estimate the field necessary to align dipoles with a different dipole moment $\boldsymbol{p}$ by:

$$
E_{\mathrm{th}} \approx 20 \frac{k_{\mathrm{B}} T}{\boldsymbol{p}}
$$

\section{Conclusions}

We have shown that colloidal CdSe/CdS NRs can be fully aligned in a colloidal dispersion by the application of AC electric fields with an appropriate field strength. The alignment is monitored by measuring the variation of the transmission concurring with the oscillation of the electric field at wavelengths where the CdS rod absorption dominates. For the rods used, complete alignment parallel with the electric field is obtained for field strengths exceeding $15 \mathrm{~V} \mathrm{\mu m}^{-1}$ and switching back and forth to random orientation occurs within $50 \mu \mathrm{s}$. We develop a theoretical description of the relation between the electric field and the alignment of dielectric ellipsoidal nano-objects, considering both a permanent and an induced dipole moment. Doing so, it follows that the experimentally determined alignment as a function of field strength can be attributed to the interaction between the NR permanent dipole and the applied AC field.

Since the transmission change in the regime of full alignment only depends on the difference between the NR absorption coefficient for optical fields parallel and perpendicular to the NR long axis, transmission measurements during AC driven full alignment provide an excellent method to measure anisotropic absorption by NRs. Excellent agreement between experiment and theory is obtained for the absorption anisotropy at $470 \mathrm{~nm}$, i.e., wavelengths where the rod absorption dominates. Moreover, we find that band-edge transition exhibits a quite similar absorption anisotropy, a result we attribute to the small dielectric mismatch between the CdSe core and the CdS rod.

\section{Acknowledgements}

This research was supported by the Interuniversity Attraction Poles program of the Belgian Science Policy Office, under grant IAP P7-35.

\section{References}

1 E. R. Smith, J. M. Luther and J. C. Johnson, Nano Lett., 2011, 11, 4923-4931.

2 J. Cao, T. Sun and K. T. V. Grattan, Sens. Actuators, B, 2014, 195, 332-351.

3 M. Shim and H. McDaniel, Curr. Opin. Solid State Mater. Sci., 2010, 14, 83-94.

4 Z. Hens and I. Moreels, J. Mater. Chem., 2012, 22, 1040610415.

5 F. Pisanello, L. Martiradonna, P. Spinicelli, A. Fiore, J. P. Hermier, L. Manna, R. Cingolani, E. Giacobino,
A. Bramati, M. De Vittorio and Ieee, Polarized Single Photon Emission for Quantum Cryptography Based on Colloidal Nanocrystals, Ieee, New York, 2009.

6 R. Krahne, G. Morello, A. Figuerola, C. George, S. Deka and L. Manna, Phys. Rep., 2011, 501, 75-221.

7 K. Becker, J. M. Lupton, J. Muller, A. L. Rogach, D. V. Talapin, H. Weller and J. Feldmann, Nat. Mater., 2006, 5, 777-781.

8 W. S. Chang, J. W. Ha, L. S. Slaughter and S. Link, Proc. Natl. Acad. Sci. U. S. A., 2010, 107, 2781-2786.

9 A. Rizzo, C. Nobile, M. Mazzeo, M. De Giorgi, A. Fiore, L. Carbone, R. Cingolani, L. Manna and G. Gigli, ACS Nano, 2009, 3, 1506-1512.

10 M. Fisher, M. Zanella, D. J. Farrell, L. Manna, P. Stavrinou and A. J. Chatten, 2011 37th IEEE Photovoltaic Specialists Conference (PVSC 2011), 2011, pp. 000858-000863.

11 C. Nobile, V. A. Fonoberov, S. Kudera, A. Della Torre, A. Ruffino, G. Chilla, T. Kipp, D. Heitmann, L. Manna, R. Cingolani, A. A. Balandin and R. Krahne, Nano Lett., 2007, 7, 476-479.

12 F. Liu, J. Wang, Z. Ge, K. Li, H. Ding, B. Zhang, D. Wang and H. Yang, J. Mater. Chem. C, 2013, 1, 216-219.

13 A. Persano, M. De Giorgi, A. Fiore, R. Cingolani, L. Manna, A. Cola and R. Krahne, ACS Nano, 2010, 4, 1646-1652.

14 A. Singh, R. D. Gunning, S. Ahmed, C. A. Barrett, N. J. English, J. A. Garate and K. M. Ryan, J. Mater. Chem., 2012, 22, 1562-1569.

15 C. Querner, M. D. Fischbein, P. A. Heiney and M. Drndic, Adv. Mater., 2008, 20, 2308.

16 A. Ghezelbash, B. Koo and B. A. Korgel, Nano Lett., 2006, 6, 1832-1836.

17 D. V. Talapin, E. V. Shevchenko, C. B. Murray, A. Kornowski, S. Forster and H. Weller, J. Am. Chem. Soc., 2004, 126, 1298412988.

18 M. Zanella, R. Gomes, M. Povia, C. Giannini, Y. Zhang, A. Riskin, M. Van Bael, Z. Hens and L. Manna, Adv. Mater., 2011, 23, 2205.

19 J. L. Baker, A. Widmer-Cooper, M. F. Toney, P. L. Geissler and A. P. Alivisatos, Nano Lett., 2010, 10, 195-201.

20 Y. Amit, A. Faust, I. Lieberman, L. Yedidya and U. Banin, Phys. Status Solidi A, 2012, 209, 235-242.

21 Z. H. Hu, M. D. Fischbein, C. Querner and M. Drndic, Nano Lett., 2006, 6, 2585-2591.

22 K. M. Ryan, A. Mastroianni, K. A. Stancil, H. T. Liu and A. P. Alivisatos, Nano Lett., 2006, 6, 1479-1482.

23 M. Mohammadimasoudi, L. Penninck, T. Aubert, R. Gomes, Z. Hens, F. Strubbe and K. Neyts, Opt. Mater. Express, 2013, 3, 2045-2054.

24 A. Singh, N. J. English and K. M. Ryan, J. Phys. Chem. B, 2013, 117, 1608-1615.

25 D. Baranov, A. Fiore, M. van Huis, C. Giannini, A. Falqui, U. Lafont, H. Zandbergen, M. Zanella, R. Cingolani and L. Manna, Nano Lett., 2010, 10, 743-749.

26 N. Zhao, K. Liu, J. Greener, Z. H. Nie and E. Kumacheva, Nano Lett., 2009, 9, 3077-3081.

27 A. M. Hung, N. A. Konopliv and J. N. Cha, Langmuir, 2011, 27, 12322-12328. 
28 A. M. Hung, T. Oh and J. N. Cha, Nanoscale, 2012, 4, 10161020.

29 F. Pietra, F. T. Rabouw, P. G. van Rhee, J. van Rijssel, A. V. Petukhov, B. H. Erné, P. C. M. Christianen, C. de Mello Donegá and D. Vanmaekelbergh, ACS Nano, 2014, 8, 10486-10495.

30 L. S. Li and A. P. Alivisatos, Phys. Rev. Lett., 2003, 90, 097402. 31 J. S. Kamal, R. Gomes, Z. Hens, M. Karvar, K. Neyts, S. Compernolle and F. Vanhaecke, Phys. Rev. B: Condens. Matter Mater. Phys., 2012, 85, 035126.

32 H. E. Ruda and A. Shik, Nanotechnology, 2010, 21, 235502.
33 R. Krishnan, M. A. Hahn, Z. H. Yu, J. Silcox, P. M. Fauchet and T. D. Krauss, Phys. Rev. Lett., 2004, 92, 216803.

34 L. D. Landau, E. M. Lifshitz and E. Mikhailovich, Electrodynamics of continuous media, Oxford, 1984, p. 331.

35 A. Sihvola, J. Nanomater., 2007, 45090.

36 G. Volpe and D. Petrov, Phys. Rev. Lett., 2006, 97, 210603.

37 D. Ricard, M. Ghanassi and M. C. Schanneklein, Opt. Commun., 1994, 108, 311-318.

38 M. Cirillo, F. Strubbe, K. Neyts and Z. Hens, ACS Nano, 2011, $5,1345-1352$. 